\title{
Zentraler Einfluss des Alters und Apolipoprotein-E-Genotyps
}

Fragestellung: Die Studie untersucht, in welcher Weise das Personenalter und der Apolipoprotein-E(ApoE)-Genotyp die Prävalenz zerebraler Ablagerungen von $\beta$-Amyloid $(\mathrm{A} \beta)$ bei kognitiv Gesunden und Patienten mit Demenz beeinflussen.

Hintergrund: Mittels geeigneter Positronen-Emissions-Tomografie(PET)-Tracer ist es möglich, zerebrale $A \beta$-Ablagerungen nicht-invasiv im lebenden Gehirn nachzuweisen. Studien deuten darauf hin, dass das Auftreten zerebraler A $\beta$-Pathologie der Entwicklung des Morbus Alzheimer um mindestens 20 Jahre vorausgeht. Valide Untersuchungen zu Alters- und ApoE-Genotyp-Einflüssen auf das Vorhandensein zerebraler A $\beta$-Pathologie bei kognitiv Gesunden und Demenzpatienten fehlten.

Patienten und Methodik: Eine Metaanalyse mit 2.914 Teilnehmern mit unauffälliger Kognition, 697 mit subjektiver sowie 3.972 mit leichter kognitiver Beeinträchtigung, 1.359 mit klinisch diagnostiziertem Morbus Alzheimer und 538 mit Nicht-

Jansen WJ, Ossenkoppele R, $\mathrm{Knol}$ DL et al. Prevalence of cerebral amyloid pathology in persons without dementia: a metaanalysis. JAMA 2015; 313: 1924-38

Ossenkoppele R, Jansen WJ, Rabinovici GD et al. Prevalence of amyloid PET positivity in dementia syndromes: a meta-analysis. JAMA 2015; 313: 1939-49
Alzheimer-Demenz untersuchte den Einfluss des Alters und des ApoE-Genotyps auf eine mittels PET nachweisbare A $\beta$-Pathologie. Voraussetzung für den Studieneinschluss war die Verwendung A $\beta$-spezifischer Tracer. 1.369 Patienten mit autoptisch gesichertem Morbus Alzheimer dienten als weitere Kontrollgruppe.
Ergebnisse: Bei Personen mit normaler kognitiver Leistungsfähigkeit stieg die Prävalenz einer mittels PET nachweisbaren A $\beta$-Pathologie altersabhängig von $10 \%$ (95\%-Konfidenzintervall $8-13 \%)$ im Alter von 50 Jahren auf $44 \%$ (37 - 51\%) im Alter von 90 Jahren. Bei subjektiver kognitiver Beeinträchtigung stieg die Prävalenz im gleichen Altersintervall von $12 \%(8-18 \%)$ auf $43 \%(32-55 \%)$ und bei leichter kognitiver Beeinträchtigung von $27 \%(23-32 \%)$ auf $71 \%(66-76 \%)$. ApoE- $\varepsilon 4$-Carrier hatten eine zwei- bis dreimal höhere Prävalenz als ApoE- $\varepsilon 4-N o n-$ Carrier. Bei Patienten mit Morbus Alzheimer hingegen sank die Prävalenz der A $\beta$-Pathologie bei ApoE- $\varepsilon 4-N o n-C a r r i e r n$ von $86 \%(73-94 \%)$ im Alter von 50 Jahren auf $68 \%$ (57-77\%) im Alter von 90 Jahren und bei ApoE- $\varepsilon 4$-Carriern von $97 \%$ (92-99\%) im Alter von 50 Jahren auf $90 \%$ (83-94\%) im Alter von 90 Jahren. Ähnliche Assoziationen von Alter und ApoEGenotyp mit dem Vorhandensein der A $\beta$-Pathologie wurden histologisch bei autoptisch gesichertem Morbus Alzheimer beobachtet. Bei Patienten mit Nicht-Alzheimer-Demenz (frontotemporale Demenz, vaskuläre Demenz, Lewy-Körperchen-Erkrankung) wiederum stieg die Prävalenz der A $\beta$-Pathologie altersabhängig in Abhängigkeit vom ApoE-Genotyp an.

Schlussfolgerungen: Altersabhängige Prävalenzen unterstützen die Annahme, dass das Auftreten der A $\beta$-Pathologie der Entwicklung des klinisch manifesten Morbus Alzheimer um 20 bis 30 Jahre vorausgeht. Die Daten belegen den Nutzen des A $\beta$ Imaging bei der Differenzialdiagnose im früheren Lebensalter auftretender Demenzerkrankungen bei ApoE- $\varepsilon 4$-Carriern und -Non-Carriern und im späteren Lebensalter auftretender

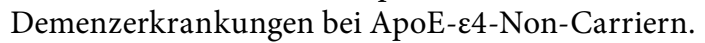

\section{- Kommentar von Dirk Hermann, Essen}

\section{Demenz im hohen Alter oft nicht mit $\beta$-Amyloid-Pathologie assoziiert}

Die vorgelegte Studie präsentiert erstmals verlässliche Zahlen zur Prävalenz der mittels PET nachweisbaren A $\beta$-Pathologie bei kognitiv Gesunden und Demenzpatienten. Auffällig ist der starke Einfluss des ApoE-Genotyps auf die Prävalenz der A $\beta$ Pathologie, die den klinisch tätigen Neurologen vor das Dilemma stellt, das lebenszeitabhängige Risiko zuverlässig einschätzen zu können, ohne kausale Therapien anbieten zu können, die erlauben, das Alzheimer-Risiko zu senken.

Das altersabhängige Absinken des Nachweises einer $A \beta$ Pathologie bei Patienten mit klinisch diagnostiziertem Morbus Alzheimer wirft die Frage nach der korrekten Klassifikation des Morbus Alzheimer im hohen Lebensalter auf. Die Demenzdiagnose im hohen Lebensalter ist erschwert durch eingeschränkte Alltagsaktivitäten, weshalb kognitive Defizite oft nicht zuverlässig beurteilbar sind. Zukünftige Studien sollten der Frage nachgehen, ob Patienten, bei denen fälschlicherweise eine $A \beta$-Pathologie unterstellt wird, in ähnlicher Weise wie Patienten mit $A \beta$-Pathologie von einer Behandlung ihrer Demenzerkrankung profitieren.

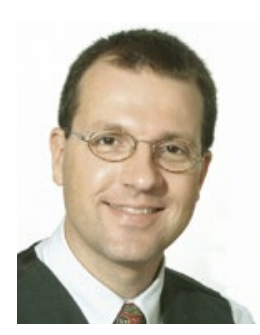

Prof. Dr. med. Dirk M. Hermann, Essen

Lehrstuhl für vaskuläre Neurologie, Demenz \& Altersforschung, Universitätsklinikum Essen E-Mail: dirk.hermann@uk-essen.de 